\title{
Humanizing malaria mice
}

\section{By Tim Fulmer, Senior Writer}

U.S. researchers have developed the first mouse model that recapitulates the human stages of the malarial parasite Plasmodium falciparum life cycle in one animal. ${ }^{1}$ The academics plan to make additional modifications to the mice to improve their utility, but pharmas already want to use the animals in early stage research.

The life cycle of $P$. falciparum has three distinct stages. The liver and blood stages occur in the host, whereas the sexual stage occurs in the mosquito gut.

During the asymptomatic liver stage, sporozoites migrate from the mosquito bite site to the host's liver, where they form merozoites within liver cells. In the blood stage, the merozoites enter circulation and invade erythrocytes, which rupture and release new merozoites and gametocytes. The latter then are transmitted to a mosquito during feeding.

Because P. falciparum only infects humans and primates, studying the parasite in rodents has been impossible, leaving researchers with only two options for early stage in vivo studies of the disease.

One is to use malarial strains such as $P$. berghei and P. yoelii that infect rodents as proxies for P. falciparum. ${ }^{2}$ Although this has allowed the study of both host stages in the same animal, rodent strains are evolutionarily distant from $P$. falciparum, and as a result those strains lack many $P$. falciparum proteins that could be therapeutic targets or vaccine candidates in humans.

The second option is to humanize mice to make them susceptible to $P$. falciparum infection. This commonly involves engrafting human red blood cells (RBCs) or hepatocytes into immunocompromised mice. ${ }^{3}$ Although separate humanized models of blood-stage and liver-stage infection exist, ${ }^{4,5}$ no humanized models combining both liver and blood stages have been disclosed.

Researchers at the Seattle Biomedical Research Institute, The Rockefeller University and Yecuris Corp. decided to develop a humanized malaria mouse model that develops liver-stage $P$. falciparum infection and transitions to blood-stage infection.

Yecuris was founded in 2007 to commercialize transgenic mouse technology developed by Markus Grompe and colleagues at the Oregon Health \& Science University.

As a starting point for the new malaria model, the researchers used one of the mouse models marketed by Yecuris-the FRG (FahRag2-Cd132) triple-knockout mouse. ${ }^{6,7}$

In FRG mice, knocking out recombination activating gene 2 (Rag2) and Il-2 receptor $\gamma$-chain (Cd132) leads to severe immunodeficiency, whereas knocking out fumarylacetoacetate hydrolase (Fah) leads to death of hepatocytes from excess buildup of the metabolite fumarylacetoacetate. The resulting animals are then engrafted with functional human hepatocytes to generate animals with human liver tissue.

Until now, FRG mice have primarily served as models of human liver disease and hepatitis infection. However, the researchers hypothesized that it might be possible to establish a liver-stage $P$. falciparum infection in the FRG mice as a first step in the development of a two-stage model of human malaria infection.

To test that idea, the researchers injected $F R G$ mice intravenously with mosquito salivary gland P. falciparum sporozoites and euthanized the animals three, five, six and seven days postinfection. At each time point, liver tissue was analyzed for signs of progression of liver-stage P. falciparum infection.

At day three, liver-stage parasites were detectable in the engrafted human hepatocytes. Between days three and seven, the parasites developed into schizonts, which are mature forms of the parasite that contain merozoites. At day seven, aggregates of merozoites budded off from the liver cells into surrounding tissues, completing the liver stage.

Next, the researchers set out to test whether the FRG mice would also allow the engraftment of human RBCs to study the blood stage of P. falciparum infection.

A first round of experiments showed that the $F R G$ mice rapidly cleared engrafted human RBCs, suggesting that additional genetic modifications would be required. The researchers backcrossed the FRG mice with nonobese diabetic (NOD) mice, a strain that typically shows reduced clearance of engrafted human RBCs.

The resulting FRG NOD mice were injected intravenously with human RBCs at six days post-sporozoite injection. Analysis of blood removed on day seven revealed blood-stage parasites that grew in culture at a rate similar to that of the parent $P$. falciparum strain used to generate the initial sporozoites.

Thus, the FRG NOD mice supported the establishment of the liver stage of $P$. falciparum infection and the transition of the disease from liver stage to blood stage.

The findings were published in The Journal of Clinical Investigation.

"The key advance in the present study is that they obtained the complete transition from the $P$. falciparum hepatic stages to infected red blood cells in vivo in the same mouse," said Dominique Mazier. "The model opens the way to assess the effect of a drug throughout hepatic development including in the presence of infected erythrocytes, and this is a clear step forward."

Mazier and colleagues "The model opens the way to assess the effect of a drug throughout hepatic development including in the presence of infected erythrocytes, and this is a clear step forward." -Dominique Mazier,

Pierre and Marie Curie University infected primary human hepatocyte cultures for compound screening. ${ }^{8}$ Mazier is professor of parasitology and mycology at the Pierre and 
Marie Curie University and head of the malaria drug and vaccine target identification laboratory at Institut National de la Santé et de la Recherche Médicale (INSERM).

Other potential uses of the FRG NOD mice, according to Iñigo AnguloBarturen, include "looking at the effects of mutations on the liver-stage development of the parasite as well as doing proteomic/metabolomic analysis of the parasite under different physiological conditions." Angulo-Barturen is chief scientist of the Therapeutic Efficacy unit at GlaxoSmithKline plc.

\section{Next steps}

"We are already discussing with companies, including Novartis and GSK, how our mice might be used to test the efficacy of antimalarial compounds," corresponding author Stefan Kappe told SciBX.

Kappe is professor and malaria program director at the Seattle Biomedical Research Institute and affiliate professor of global health at the University of Washington.

Indeed, GSK "hopes to set up the new model in-house, or an adapted version of it useful for drug discovery, in order to test compounds that kill liver stages of P. falciparum and, hopefully, P. vivax," said AnguloBarturen. $P$. vivax "produces quiescent liver stages that can be present in liver tissues for months or years until reactivation provokes a new malaria episode."

The mice could replace the current nonhuman primate model of $P$. vivax infection, said Tim Wells, CSO of Medicines for Malaria Venture (MMV).

Much of the focus of both GSK and MMV has been on developing compounds that target the blood stages of the parasite.

Since the mid-1990s, GSK has collaborated with Leonard Shultz and colleagues at The Jackson Laboratory to develop two humanized mouse models of blood-stage P. falciparum infection. The models are based on NOD-SCID mice that are deficient in either Cd132 or $\beta_{2}$ microglobulin (B2m). ${ }^{9} 10$ The resulting animals are regularly injected with human RBCs and used for testing the efficacy of antimalarial compounds, according to Angulo-Barturen.

MMV primarily uses cell-based phenotypic screens to look at the effects of compounds directly on the malaria parasite and has screened over five million compounds over the past five years, said Wells.

He added that the most advanced compound to result from the screen is the spiroindolone-based molecule NITD609, ${ }^{11,12}$ which is being developed by Novartis AG in collaboration with MMV. The compound entered Phase II testing this year.

Novartis did not respond to requests for comment.

"The mice will be useful for doing genetic crosses of multiple P. falciparum strains to identify genomic modifications in the parasite that confer drug resistance," said Kappe.

"Also, we should be able to use the mice to measure the attenuation levels of $P$. falciparum sporozoites used in vaccines," said Alexander Ploss, a principal investigator on the JCI paper. "This will allow us to better determine whether a given strain is attenuated at sufficiently safe levels before testing it in human volunteers." Ploss is assistant professor of virology and infectious disease at Rockefeller University.

Finally, additional work can still be done on the mice to establish a more robust blood-stage infection, said Ploss.

"There are two general ways we are considering doing that," he said. "One approach is basically to combine the liver chimeric mouse we have now with the humanized blood-stage mice used by GSK and others. Another, more ambitious approach is to modify our mice into a double transplant model, where we transplant not only human hepatocytes for liver-stage studies but also human hematopoietic stem cells for blood-stage studies. In that scenario, the animals don't require regular injections of human RBCs but instead endogenously produce the entire human erythrocyte lineage."

Ploss said he and Kappe are collaborating with Richard Flavell to develop those mouse models. Flavell is professor of immunology at the Yale School of Medicine.

The FRG and FRG NOD mice used in the JCI paper are covered by patents and are available for purchase from Yecuris.

Fulmer, T. SciBX 5(38); doi:10.1038/scibx.2012.997

Published online Sept. 27, 2012

\section{REFERENCES}

1. Vaughan, A.M. et al. J. Clin. Invest.; published online Sept. 10, 2012; doi:10.1172/JCl62684

Contact: Stefan H.I. Kappe, Seattle Biomedical Research Institute, Seattle, Wash. e-mail: stefan.kappe@seattlebiomed.org

2. Langhorne, J. et al. Malar. J. 10, 23 (2011)

3. Vaughan, A.M. et al. Future Microbiol. 7, 657-665 (2012)

4. Sacci, J.B. Jr. et al. Int. J. Parasitol. 36, 353-360 (2006)

5. VanBuskirk, K.M. et al. Proc. Natl. Acad. Sci. USA 106, 1300413009 (2009)

6. Azuma, H. et al. Nat. Biotechnol. 25, 903-910 (2007)

7. Bissig, K.-D. et al. Proc. Natl. Acad. Sci. USA 104, 20507-20511 (2007)

8. Mazier, D. et al. Nat. Rev. Drug Discov. 8, 854-864 (2009)

9. Angulo-Barturen, I. et al. PLoS ONE 3, e2252; published online May 21, 2008; doi:10.1371/journal.pone.0002252

10. Jiménez-Díaz, M.B. et al. Antimicrob. Agents Chemother. 53, 4533-4536 (2009)

11. Rottmann, M. et al. Science 329, 1175-1180 (2010)

12. Yeung, B.K.S. et al. J. Med. Chem. 53, 5155-5164 (2010)

COMPANIES AND INSTITUTIONS MENTIONED GlaxoSmithKline plc (LSE:GSK; NYSE:GSK), London, U.K. Institut National de la Santé et de la Recherche Médicale, Paris, France

The Jackson Laboratory, Bar Harbor, Maine

Medicines for Malaria Venture, Geneva, Switzerland

Novartis AG (NYSE:NVS; SIX:NOVN), Basel, Switzerland

Oregon Health \& Science University, Portland, Ore.

Pierre and Marie Curie University, Paris, France

The Rockefeller University, New York, N.Y.

Seattle Biomedical Research Institute, Seattle, Wash.

University of Washington, Seattle, Wash.

Yale School of Medicine, New Haven, Conn.

Yecuris Corp., Portland, Ore. 